\title{
Implementación de la metodología del contrato de aprendizaje en la totalidad de una materia de fisioterapia: opinión de los alumnos
}

\author{
Gustavo Rodríguez-Fuentes, Iris Machado de Oliveira
}

Introducción. En la metodología de contrato de aprendizaje existe una participación activa de profesores y estudiantes, algo que se considera crucial para promover un adecuado aprendizaje en los días actuales.

Objetivo. Conocer la opinión de los alumnos tras la aplicación de la metodología del contrato de aprendizaje para el desarrollo completo de una materia del Título de Grado de Fisioterapia de la Universidad de Vigo.

Sujetos y métodos. Estudio descriptivo transversal postintervención con todos los alumnos matriculados en la materia. Opinión recogida mediante un cuestionario elaborado ad hoc.

Resultados. El alumnado resaltó, de forma positiva, su opción libre por el contrato de aprendizaje, el apoyo recibido del profesorado a lo largo de todo el proceso, la realización y entrega de las evidencias exigidas, el beneficio del trabajo realizado para superar la materia, el respeto por los criterios de evaluación firmados, el proceso de evaluación desarrollado, la consideración como justa de la calificación final obtenida y la alta valoración del profesorado. De forma negativa se ha mencionado la mayor carga de trabajo en comparación con la metodología tradicional.

Conclusiones. El contrato de aprendizaje parece una buena estrategia docente de cara a facilitar la participación activa de los alumnos en su propio aprendizaje. Es un aspecto de gran importancia en los profesionales de la salud para desarrollar las competencias que faciliten un aprendizaje autónomo a lo largo de su vida.

Palabras clave. Aprendizaje. Educación superior. Enseñanza. Fisioterapia. Modelos educacionales.

Facultad de Fisioterapia. Universidad de Vigo. Pontevedra, España.

Correspondencia: Dra. Iris Machado de Olivera. Facultad de Fisioterapia. Universidad de Vigo. Campus A Xunqueira, $\mathrm{s} / \mathrm{n}$ E-36005 Pontevedra. Fax: +34986801780.

E-mail: irismacoli@uvigo.es

Conflicto de intereses: No declarado.

Competing interests: None declared.

(c) $2014 \mathrm{FEM}$

The implementation of the learning contract methodology in a entire course of Physiotherapy: student's opinion

Introduction. In learning contract methodology there is a significantly active involvement of teachers and students, something crucial in promoting a proper learning nowadays.

Aim. To know the students' opinion on the implementation of the learning contract methodology in a course of the Physiotherapy Degree at University of Vigo.

Subjects and methods. A cross-sectional post-intervention descriptive study was carried out with all enrolled students in the course. The students' opinion was gathered through an ad-hoc questionnaire.

Results. The most positive outcomes of our study were the warm approach provided by the teacher for students' individual consults, the teacher's concern to answering personally and in writing all the doubts, the consideration by the students that all the work carried out helped them to understand the topics and consequently to pass the course, the evaluation of the work done by the students was consistent with the criteria established by the learning contract, the evaluation of the practical part of the course matched the learning contract criteria, and that the course evaluation methods seemed equitable, adequate and consistent with the objectives of the course and with the emphasis placed by the teacher.

Conclusions. The learning contract seems to be a good teaching strategy used to facilitate the student's active participation in their own learning ability. This is a very important point to help the future health professionals to improve their skills and to develop the capacity of learning throughout their professional career.

Key words. Educational models. Higher education. Learning. Physiotherapy. Teaching. 


\section{Introducción}

Estar expuesto a una variedad de estrategias de enseñanza y aprendizaje es muy importante para todos los estudiantes en los diferentes niveles educativos $[1,2]$. En la actualidad, existen enfoques pedagógicos en educación superior que se basan en el contenido y se centran en la figura del profesor, y otros basados en la figura del estudiante y centrado en sus necesidades [3].

El uso de nuevas estrategias de aprendizaje en la educación superior como, por ejemplo, el aprendizaje basado en problemas o el aprendizaje cooperativo, está muy valorado en la enseñanza universitaria de las ciencias de la salud [4], pero estas estrategias dependerán en cuanto a la percepción de los estudiantes, en cierta medida, del contexto académico y de la importancia de cada materia específica en la que se incluyan en los diferentes grados [1].

Considerar un enfoque de alumno más activo, como podría suceder en el Grado en Fisioterapia, facilitaría implementar una amplia variedad de herramientas relevantes para su futuro desempeño profesional, un futuro profesional donde se les pedirá que desarrollen su función clínica en un contexto complejo y en constante cambio [2]. Una de esas estrategias que facilitan el aprendizaje activo de los alumnos es el contrato de aprendizaje, desarrollado por Knowles [5] y que constituye una concreción de los denominados 'contratos conductuales' [6].

El contrato de aprendizaje consiste en la firma de un acuerdo entre profesor y alumno. Dicho acuerdo define los objetivos de aprendizaje, recursos y estrategias a emplear, temporalización, evidencias a presentar y la metodología de evaluación $[5,7,8]$. Por lo tanto, el contrato de aprendizaje implica el desarrollo de un proceso educativo centrado en el estudiante [2,5,9-11], proceso que promueve el autoaprendizaje $[9,11-13]$ y que se asemeja a la forma en que todo profesional debe actuar cuando se enfrenta a un nuevo conocimiento a lo largo de su vida $[9,11,14-16]$. Esta forma de trabajo debe considerarse como un tipo de aprendizaje activo, aspecto que se destaca en las directrices del Espacio Europeo de Educación Superior. En este nuevo contexto educativo, el estudiante ya no es un simple receptor pasivo de conocimiento, como ocurre en la metodología de enseñanza-aprendizaje tradicional, sino un participante activo en la construcción de su propio conocimiento.

El contrato de aprendizaje es una herramienta pedagógica utilizada con frecuencia en los programas de formación práctico-clínica en ciencias de la salud [2,7,9-12,15,17-23]. Sin embargo, en este es- tudio, se estableció para una materia teórico-práctica del Grado en Fisioterapia de la Universidad de Vigo, concretamente para 'Electroterapia y ultrasonoterapia' (materia del segundo semestre del segundo curso de la titulación), cuya denominación, en la diplomatura (título antiguo) era 'Fisioterapia general III', aunque tenía los mismos contenidos (por eso, a partir de aquí sólo hablaremos de 'Electroterapia y ultrasonoterapia'). La justificación del uso del contrato de aprendizaje vino determinada por las especiales condiciones que se dieron en su momento:

- En la Universidad de Vigo, en el curso 2009-2010, se produjo una transformación simultánea de todos los cursos del Título de Diplomado en Fisioterapia (tres años de duración) al Título de Grado en Fisioterapia (cuatro años), dejando elegir a los alumnos que no fuesen de primera matrícula entre seguir con el plan de estudios antiguo o cambiarse al nuevo.

- En relación a la materia 'Electroterapia y ultrasonoterapia, señalar que consta de 6 créditos ECTS (European Credit Transfer System), que se traducen en 50 horas de clase presencial (18 horas teóricas, 30 horas prácticas y 2 horas para la exposición de un trabajo académico) y 100 horas de trabajo personal (preparar el contenido teórico y práctico de la materia, realizar el trabajo y preparar los exámenes).

- Dada la transformación simultánea de cursos de un título al otro en el año académico 2009-2010 y a que la materia cambió de curso su impartición, se dio la circunstancia de que los alumnos matriculados ese año en 'Electroterapia y ultrasonoterapia' fueron sólo los que no habían aprobado el año anterior, es decir, siete matriculados (cuatro de ellos optaron por el nuevo Título de Grado).

- Teniendo en cuenta el pequeño número de matriculados y que ya habían cursado la materia el curso anterior, se optó por plantear al alumnado el desarrollo de toda la materia a través de un contrato de aprendizaje, metodología educativa desarrollada para grupos de un máximo 12-14 alumnos [10,17] y que, además, presenta otros posibles beneficios señalados en la bibliografía $[2,9,12,21]$ : motiva el aprendizaje del alumno al constituirlo en actor activo de éste, facilita el desarrollo de un aprendizaje significativo adquiriendo al mismo tiempo una herramienta útil para su futuro, fomenta el compromiso en el proceso de enseñanza-aprendizaje, hay unas normas explícitas que asumen tanto docentes como alumnado y, por ejemplo, facilita la evaluación de los implicados en el proceso de enseñanza- 
aprendizaje, así como del propio proceso. El hecho de que los estudiantes matriculados ya habían cursado la asignatura el año académico anterior significaba que ellos conocían los objetivos, competencias y contenidos de ésta, además de saber cuáles habían sido sus problemas en el proceso de aprendizaje y qué les había conducido a no superar la materia.

Para poner en marcha este proyecto de innovación educativa, se siguieron los seis pasos descritos por Bailey y Tuohy [2], los cuales coinciden con los requisitos básicos que todo contrato de aprendizaje debe contener según Anderson et al [24] y Knowles [5], y que son:

- Identificación de las necesidades de aprendizaje.

- Especificación de los objetivos de aprendizaje.

- Identificación de recursos y estrategias necesarios para alcanzar dichos objetivos.

- Determinación de qué serán evidencias de logro de dicho aprendizaje y de cómo se valorarán.

- Desarrollo del contrato de aprendizaje tras su firma por el profesor y por el alumno.

- Evaluación del aprendizaje y del proceso seguido.

El objetivo de este estudio fue conocer la opinión de los alumnos tras la aplicación de la metodología del contrato de aprendizaje para el desarrollo completo de la materia teórico-práctica de 'Electroterapia y ultrasonoterapia, impartida en el Título de Grado de Fisioterapia de la Universidad de Vigo.

\section{Sujetos y métodos}

Se realizó un estudio descriptivo transversal postintervención. El estudio estuvo vinculado al proyecto educativo de transformación simultánea de todos los cursos de la Diplomatura de Fisioterapia al Grado de Fisioterapia, siendo aprobada por la Facultad de Fisioterapia de la Universidad de Vigo (acta n. $107 / 2009)$.

Inicialmente, la muestra estuvo compuesta por todos los alumnos matriculados en la materia 'Electroterapia y ultrasonoterapia' $(n=7)$ del Título de Grado de Fisioterapia de la Universidad de Vigo en el curso académico 2009-2010. Dos semanas después del inicio del semestre académico, una estudiante abandonó los estudios, por lo que el número final de alumnos matriculados fue de seis.

El grupo de alumnos y el desarrollo de la materia fue llevado por un único profesor (G.R.F.). En la interpretación de los datos y la elaboración del manuscrito también participó activamente otra profe-
Figura. Condiciones del contrato de aprendizaje (CA) seguido para la materia 'Electroterapia y ultrasonoterapia' de la Universidad de Vigo.

\begin{tabular}{|c|c|c|}
\hline \multicolumn{3}{|c|}{ Objetivos generales del proceso de enseñanza-aprendizaje a través del CA } \\
\hline Conocimientos & Habilidades y destrezas & Actitudes y valores \\
\hline $\begin{array}{l}\text { Conocer, comprender y analizar las } \\
\text { bases teóricas y prácticas que } \\
\text { fundamentan el uso terapéutico de la } \\
\text { corriente eléctrica y los ultrasonidos. } \\
\text { Conocer, comprender y analizar el } \\
\text { procedimiento de valoración y aplicación } \\
\text { de unas corrientes eléctricas o de unos } \\
\text { ultrasonidos terapéuticos en un caso } \\
\text { clínico. }\end{array}$ & $\begin{array}{l}\text { Adquirir la destreza necesaria para } \\
\text { el manejo y aplicación de unos } \\
\text { equipos de electroterapia y } \\
\text { ultrasonoterapia, así como de } \\
\text { manejo de un futuro paciente. } \\
\text { Mejorar las habilidades de } \\
\text { comunicación con los demás, asi } \\
\text { como la defensa de las propias } \\
\text { ideas. }\end{array}$ & $\begin{array}{l}\text { Fomentar un espiritu critico, la confianza } \\
\text { en uno mismo y la toma de decisiones. } \\
\text { Fomentar el respeto a las opiniones del } \\
\text { otro (alumnos o profesor). } \\
\text { Fomentar la responsabilidad e implicación } \\
\text { personal en el trabajo a desarrollar. } \\
\text { Evaluar el trabajo realizado por los } \\
\text { compañeros y el profesor. }\end{array}$ \\
\hline \multicolumn{3}{|c|}{ Contenidos de la materia } \\
\hline \multicolumn{3}{|c|}{$\begin{array}{l}\text { 1. Generalidades sobre electroterapia + teoria y casos prácticos de corriente galvánica continua, iontoforesis y baño galvánico. } \\
\text { 2. Tecria y casos prácticos de corrientes de baja frecuencia (excepto apartado de electrovaloración y electroestimulación neuromuscular). } \\
\text { 3. Teoría y casos prácticos de corrientes de baja frecuencia (apartado de electrovaloración y electroestimulación neuromuscular). } \\
\text { 4. Teoria y casos prácticos de corrientes de media frecuencia } \\
\text { 5. Teoria y casos prácticos de corrientes de alta frecuencia } \\
\text { 6. Teoria y casos prácticos de ultrasonoterapia y terapia combinada + Teoría sobre ondas de choque. }\end{array}$} \\
\hline \multicolumn{3}{|c|}{ Recursos ofrecidos } \\
\hline \multicolumn{3}{|c|}{$\begin{array}{l}\text { - Apuntes en formato Word de alumnos del curso anterior (teoria). - Estrategias de búsqueda de información en beses de datos. } \\
\text { - Apuntes en formato Word de alumnos del curso anterior (orácticas). } \\
\text { - Esquemas de los temas elaborados por el profesor. }\end{array}$} \\
\hline \multicolumn{3}{|c|}{ Cronograma del CA } \\
\hline General del semestre & & Por cada unidad didáctica \\
\hline Inicio materia: 4 de febrero de $2010 \rightarrow$ & $\begin{array}{l}\text { Explicación del CA por parte del } \\
\text { profesorado. }\end{array}$ & $\begin{array}{l}\text { - Entrega de documentos (material } \\
\text { elaborado por los propios alumnos el }\end{array}$ \\
\hline 2." semana de clase: 9 de febrero $\rightarrow$ & $\begin{array}{l}\text { Discusión, acuerdo y firma del CA } \\
+ \text { entrega de documentación para } \\
\text { la primera sesión presencial }\end{array}$ & $\begin{array}{l}\text { año anterior) y casos prácticos a los } \\
\text { alumnos (uso de la plataforma de } \\
\text { enseñanza virtual de la universidad). }\end{array}$ \\
\hline $\begin{array}{l}6 \text { sesiones presenciales }(23 \text { de febrero, } \\
9 \text { y } 23 \text { de marzo, } 13 \text { y } 27 \text { de abril, y } 11 \\
\text { de mayo) } \rightarrow\end{array}$ & $\begin{array}{l}\text { Estudio, análisis y discusión sobre } \\
\text { las evidencias a entregar, aclaraciones } \\
\text { sobre la materia (teoria y práctica) } \\
\text { y realización de casos clinicos por } \\
\text { cada una de las unidades } \\
\text { didácticas en las que se dividieron } \\
\text { los contenidos de la materia. }\end{array}$ & $\begin{array}{l}\text { - Trabajo individual no presencial de los } \\
\text { alumnos sobre el material entregado por } \\
\text { el profesor: análisis, búsqueda de } \\
\text { posibles fallos, contestación a ciertas } \\
\text { preguntas planteadas y elaboración de } \\
10 \text { preguntas tipo test. } \\
\text { - Sesión presencial: una primera parte de }\end{array}$ \\
\hline $\begin{array}{l}\text { Exámenes finales (del } 1 \text { " periodo): } \\
25 \text { de mayo de } 2010 \rightarrow\end{array}$ & $\begin{array}{l}\text { Realización de un examen teórico } \\
\text { y un examen práctico. }\end{array}$ & $\begin{array}{l}\text { análisis conjunto del trabajo realizado y } \\
\text { realización de los casos clínicos (sólo los }\end{array}$ \\
\hline Revisión de los exámenes (28 de mayo) - & $\begin{array}{l}\text { Análisis conjunta profesor-alumno } \\
\text { de la corrección de los exámenes } \\
\text { realizada }+ \text { análisis de la evaluación } \\
\text { continuada (evidencias). }\end{array}$ & $\begin{array}{l}\text { alumnos, primero, y después con el } \\
\text { profesor). Análisis y corrección de las } \\
\text { preguntas tipo test. Entrega de } \\
\text { información por parte del profesor sobre }\end{array}$ \\
\hline $\begin{array}{l}\text { Exámenes finales (del } 2 .^{\circ} \text { periodo): } \\
6 \text { de julio de } 2010 \rightarrow\end{array}$ & $\begin{array}{l}\text { Realización del examen (teórico o } \\
\text { práctico no superado en el mes de } \\
\text { mayo). }\end{array}$ & $\begin{array}{l}\text { la unidad (elaborada del propio profesor). } \\
\text { Evaluación por pares y del profesor del } \\
\text { trabajo elaborado y desarrollado por }\end{array}$ \\
\hline Revisión de los exámenes (8 de julio) $\rightarrow$ & $\begin{array}{l}\text { Análisis conjunto profesor-alumno } \\
\text { de la corrección de los exámenes } \\
\text { realizada. }\end{array}$ & cada alumno. \\
\hline Evaluación del CA (septiembre de 2010) $\rightarrow$ & $\begin{array}{l}\text { Contestación del cuestionario de } \\
\text { evaluación del CA. }\end{array}$ & \\
\hline \multicolumn{3}{|l|}{ Evidencias por cada unidad didáctica } \\
\hline - Lectura critica y corrección de los apunte & $\begin{array}{l}\text { - Elaboración } \\
\text { - Explicar y de }\end{array}$ & $\begin{array}{l}10 \text { preguntas test. } \\
\text { nostrar un caso clinico a los compañeros. }\end{array}$ \\
\hline \multicolumn{3}{|l|}{ Evaluación } \\
\hline $\begin{array}{l}\checkmark \text { Evaluación continuada (evidencias) } \\
\text { mediante rúbrica (por pares y del profesor). }\end{array}$ & $\begin{array}{l}\text { Examen teórico tipo test } \\
\text { (50 preguntas, la mitad de ellas } \\
\text { de entre las elaboradas por los } \\
\text { propios alumnos). }\end{array}$ & $\begin{array}{l}\text { Examen práctico (3 supuestos prácticos } \\
\text { elegidos al azar de entre los vistos a lo } \\
\text { largo del semestre). }\end{array}$ \\
\hline$\checkmark$ Valor: $30 \%$ del total. & Valor: $30 \%$ del total. & $\checkmark$ Valor: $40 \%$ del total. \\
\hline
\end{tabular}

sora (I.M.O.). Ambos comparten la impartición de la materia, pero por razones de número de alumnos y a que no eran primera matrícula en la transformación simultánea de la diplomatura al grado, sólo uno de los docentes tenía asignada docencia en la materia en ese año.

La metodología del estudio fue aprobada por todos los matriculados y para todo el semestre académico a través de la firma de un contrato de aprendizaje, no violando dicho contrato ningún principio ético (se les había dado como opción seguir la metodología 'tradicional' a quien quisiese). Este contrato de aprendizaje aceptaba los objetivos genera- 
Tabla I. Datos genéricos del alumnado que contestó al cuestionario de valoración del contrato de aprendizaje desarrollado.

\begin{tabular}{|c|c|c|}
\hline Sexo (mujeres/hombres) & \multicolumn{2}{|c|}{$3(60 \%) / 2(40 \%)$} \\
\hline \multirow[t]{2}{*}{ Edad media (años) } & \multicolumn{2}{|c|}{$22,2 \pm 2,86$ (rango: $20-27$ ) } \\
\hline & Bajo & 1 \\
\hline \multirow[t]{2}{*}{ Interés inicial en la materia } & Medio & 2 \\
\hline & Alto & 2 \\
\hline \multirow{2}{*}{ Materia en la que se matriculó } & FG III & 2 \\
\hline & EyU & 3 \\
\hline \multirow{4}{*}{$\begin{array}{l}\text { Grado de asistencia a las } \\
\text { fechas señaladas por el CA }\end{array}$} & $<25 \%$ & 1 \\
\hline & $25-50 \%$ & 1 \\
\hline & $50-75 \%$ & 1 \\
\hline & $>75 \%$ & 2 \\
\hline \multirow{4}{*}{$\begin{array}{l}\text { Grado de elaboración de } \\
\text { las tareas señaladas por el CA }\end{array}$} & $<25 \%$ & 0 \\
\hline & $25-50 \%$ & 0 \\
\hline & $50-75 \%$ & 2 \\
\hline & $>75 \%$ & 3 \\
\hline
\end{tabular}

Horas de trabajo o estudio dedicado a esta materia (sin contar la asistencia establecida por el CA)

Calificación final media obtenida en la materia

$6,10 \pm 1,22$ (rango: $5-8$ )

CA: contrato de aprendizaje; EyU: 'Electroterapia y ultrasonoterapia' (nombre de la materia en el grado, título actual); FG III: 'Fisioterapia general III' (nombre de la materia en la diplomatura, título antiguo).

les y las competencias a alcanzar establecidas en la guía docente de la materia, así como los contenidos básicos que en ella se señalaban, y establecía la temporalización y la evaluación a desarrollar (Figura).

La valoración realizada por los alumnos de esta experiencia se llevó a cabo a través de un cuestionario elaborado ad hoc, el cual constaba de tres partes diferenciadas. La primera, con datos genéricos. Una segunda, de 19 preguntas cerradas, que empleaba para las respuestas una escala tipo Likert de cinco opciones, desde 'totalmente en desacuerdo' (valor 1) a 'totalmente de acuerdo' (valor 5), junto a la posibilidad de no contestar. Y una tercera parte, conformada por cinco preguntas abiertas: puntos fuertes del contrato de aprendizaje como método didáctico, puntos débiles, aspectos a mejorar en la materia, aspectos a mejorar en la actuación del profesorado y, la última, para añadir cualquier otro comentario.

El cuestionario se cumplimentó en septiembre, tras finalizar la entrega de actas de la convocatoria de exámenes de julio. Para mantener el anonimato de las respuestas, se ofreció al alumnado una doble vía: a través de un formulario de Google Docs (actualmente Google Drive) o dejando el cuestionario en la plataforma de teledocencia de la Universidad de Vigo para que lo descargaran, lo cumplimentaran y lo dejaran en el buzón del profesorado.

Todos los estudiantes aceptaron participar voluntariamente en este estudio (firma de un consentimiento informado).

\section{Resultados}

Los datos, recogidos a partir de los cinco cuestionarios contestados (83,3\%), son los que se indican en las tablas I, II y III.

Dentro de las respuestas obtenidas debemos resaltar algunos datos. De forma positiva, todos los alumnos matriculados optaron libremente por el contrato de aprendizaje; en su mayoría generaron las evidencias preestablecidas en su totalidad, tuvieron una valoración positiva del apoyo recibido por parte del profesorado a lo largo de todo el proceso, del beneficio del trabajo realizado para entender y superar la materia, del cumplimiento de los criterios de evaluación firmados y del proceso de evaluación desarrollado, consideraron justa la cualificación final obtenida y dieron una alta valoración del profesorado.

En cuanto a los aspectos a mejorar, se deduce de sus respuestas que el primordial se debe a que les ha exigido mayor trabajo personal que la metodología docente 'tradicional' expositiva a cargo del profesor. Aunque reconocen al mismo tiempo que este mayor trabajo personal les facilitó superar la materia, pensaban que iba a exigirles menos esfuerzo y que les resultaría más fácil.

\section{Discusión}

Esta experiencia desarrollada con la metodología del contrato de aprendizaje ha tenido sus puntos fuertes y sus puntos débiles. En general, como primer punto fuerte, se debe resaltar un mayor predominio de percepción positiva en el alumnado, y también del profesorado, tanto por la experiencia como por el 
proceso seguido, a pesar de la novedad para ambos en su aplicación y seguimiento. Esta buena percepción, como se ha mencionado en el apartado de resultados, ha estado justificada en la opción voluntaria del alumnado por el contrato de aprendizaje, la generación de las evidencias establecidas, el apoyo recibido por parte del profesorado (rápida y adecuada respuesta a las posibles dudas que surgían y a la solución de aquellas cuestiones que no se tuvieron en cuenta al formalizar el contrato de aprendizaje), el beneficio del trabajo realizado en relación al fomento del aprendizaje en el alumnado, el proceso de evaluación desarrollado, la percepción como justa de la calificación final obtenida y la alta valoración del profesorado. Por tanto, uno de los puntos fuertes del desarrollo metodológico realizado estuvo, de modo genérico y según la percepción del alumnado, en el respeto por parte de todos los sujetos implicados por los compromisos adquiridos libremente de inicio, aspectos básicos a cumplir que señalan otros estudios [12,20,22,24].

Por otra parte, como señalan Espey et al [25], en la bibliografía se evidencia que el aprendizaje de los alumnos es más profundo con una fuerte guía por parte del profesorado, un aspecto destacado por los alumnos. Por ello, al igual que señalan otras investigaciones [2,9,10,12,14,18-20,26], consideramos que emplear el contrato de aprendizaje ha permitido desarrollar un aprendizaje de mayor calidad, pues se intentó promover en los alumnos la autonomía y el control sobre su propio aprendizaje -aspecto importante en su práctica profesional futura $[9,11,12$, $16,21]-$, aumentar su motivación por aprender y, como alguno de ellos indicó expresamente, les favoreció la aplicación de la teoría a la práctica (Tabla III, segunda respuesta de la pregunta 21). En definitiva, el contrato de aprendizaje promovió en el alumnado un aprendizaje más significativo [22]. Esto también lo manifestaron los alumnos en sus respuestas (Tabla II, pregunta 9). Como señalan Hunt et al [14], de la universidad se espera que no sólo forme a los futuros fisioterapeutas en las disciplinas específicas de la profesión, sino también en habilidades tales como pensar, comunicarse o resolver problemas [12,27], aspectos que creemos que se han visto fomentados con el contrato de aprendizaje desarrollado en esta experiencia y que suponemos que explica su uso en la formación prácticoclínica de ciencias de la salud [2,7,9-12,15,17-23].

También cabe señalar como punto fuerte la implicación del docente en el proceso, circunstancia que también se reconoce por parte de los alumnos en las respuestas al cuestionario (véase, por ejemplo, la respuesta a la pregunta 7 de la tabla II o la
Tabla II. Relación de preguntas cerradas del cuestionario y resultados de la valoración realizada por el alumnado $(n=5)$.

\begin{tabular}{lcc}
\hline & Media a & DE \\
\hline $\begin{array}{l}\text { 1. Tras las explicaciones dadas por el profesor opté libremente } \\
\text { por firmar el contrato de aprendizaje para superar la materia }\end{array}$ & 5 & 0 \\
\hline $\begin{array}{l}\text { 2. Al firmar el contrato de aprendizaje me quedaron claras las tareas } \\
\text { que debía hacer para superar la materia (lectura-corrección de apuntes, } \\
\text { preguntas test, tutorías con análisis de teoría y demostración de casos prácticos) }\end{array}$ & 4,6 & 0,9 \\
\hline $\begin{array}{l}\text { 3. Tenía claro los criterios de evaluación que se iban a emplear en la materia } \\
\text { na }\end{array}$ & 4,8 \\
\hline
\end{tabular}

4. Considero que esta metodología docente del contrato de aprendizaje a la hora de desarrollar la materia fue adecuada $4,6 \quad 0,5$

5. El material de aprendizaje dado por el profesor para preparar la materia me facilitó la elaboración de las tareas y la superación de la materia

6. Las explicaciones de la materia dadas por parte del profesor me parecieron claras

7. El profesor se mostró accesible en el trato individual con el alumnado y respondió a sus dudas tanto de manera presencial como a distancia (correo electrónico)

8. Estuvo relacionada la teoría con la práctica

$4,6 \quad 0,5$

9. Considero que el trabajo realizado (lectura, corrección de apuntes, preguntas test, análisis y demostración de casos clínicos) me ha ayudado a entender la materia y me ha facilitado su superación

\begin{tabular}{lcc}
\hline $\begin{array}{l}\text { 10. La carga de trabajo que me supuso esta materia la considero adecuada } \\
\text { 11. La evaluación del trabajo desarrollado por el alumnado se correspondió } \\
\text { con los criterios establecidos al firmar el contrato de aprendizaje }\end{array}$ & $4,8,4$ \\
\hline $\begin{array}{l}\text { 12. El examen tipo test para valorar la parte teórica de la materia } \\
\text { se correspondió con lo explicado y con el trabajo desarrollado }\end{array}$ & 4,2 & 0,8 \\
\hline $\begin{array}{l}\text { 13. La evaluación de la parte práctica de la materia se ajustó a lo firmado en } \\
\text { el contrato de aprendizaje al inicio y a lo explicado a lo largo de la materia }\end{array}$ & 5 & 0 \\
\hline $\begin{array}{l}\text { 14. Al final, la materia me pareció fácil de superar } \\
\text { 15. Los métodos de evaluación de la materia me parecieron equitativos, adecuados } \\
\text { y en correspondencia con los objetivos del curso y el énfasis puesto por el profesor }\end{array}$ & 5 & 0,5 \\
\hline $\begin{array}{l}\text { 16. El espacio docente para impartir la materia fue adecuado } \\
\text { 17. Creo que en el desarrollo de la materia hubo un buen ambiente }\end{array}$ & 4,6 & 0,5 \\
\hline $\begin{array}{l}\text { 18. La calificación obtenida en la materia me ha parecido justa } \\
\text { 19. El profesor de la materia me ha parecido un buen profesor }\end{array}$ & 5,8 & 0,4 \\
\hline
\end{tabular}

a Valores de la media: 1, totalmente en desacuerdo; 2, en desacuerdo; 3, término medio; 4, de acuerdo; 5, totalmente de acuerdo. DE: desviación estándar.

primera respuesta reflejada en la pregunta 21 de la tabla III). Realmente, este aspecto fue muy cuidado, pues la dejadez del profesorado en sus responsabili- 
Tabla III. Resultados obtenidos en la relación de preguntas abiertas del cuestionario de valoración.

20. Indica en qué se podría mejorar este método didáctico de autoaprendizaje (el contrato de aprendizaje) a la hora de desarrollar la materia:

Disponer de más horas de prácticas (1)

En vez de darnos apuntes de compañeros de años anteriores, mejor que nos diesen primero unos apuntes que no contuviesen errores y luego darnos esos otros para corregirlos nosotros (1)

No creo que sea necesario mejorar nada (3)

21. Señala cuáles fueron los aspectos más positivos que para ti tuvo este método didáctico (el contrato de aprendizaje):

Que al ser un grupo pequeño, el profesor esta siempre a la disposición de todos siempre, sin tener que esperar, con mejores explicaciones... (1)

La práctica se basó en los conocimientos propios de cada uno por lo que así nos centramos más e intentamos esforzarnos más a la hora de comprender la teoría para adecuarla a la práctica (1)

Lo más positivo: las preguntas tipo test (1)

Ninguna respuesta (2)

22. Indica qué aspectos debería mejorar el profesor:

Ninguno en especial (1)

Ninguna respuesta (4)

23. Indica qué aspectos se deberían mejorar en la materia (diferenciar entre teoría y práctica):

Que el profesor dé primero sus propios apuntes (1)

Ninguna respuesta (4)

24. Utiliza el espacio adicional para clarificar cualquiera de tus respuestas o para añadir cualquier comentario complementario:

No hubo comentarios complementarios (5)

Entre paréntesis, número de respuestas vinculadas a cada opinión reflejada.

dades docentes es una de las principales críticas que algunos autores describen al referirse al contrato de aprendizaje desde el punto de vista del profesor [20].

$\mathrm{El}$ aspecto anterior pudo verse favorecido, además, por el hecho del pequeño tamaño del grupo; la investigación sobre contrato de aprendizaje indica que no sea superior a 12-14 alumnos para un adecuado manejo del feedback y de los resultados presentados por los alumnos [10,17]. Esto, que por una parte es un aspecto positivo de cara a desarrollar el contrato de aprendizaje de forma exitosa, también establece un límite al presente estudio sobre sus posibilidades: las muestras pequeñas no permiten generalizar los resultados obtenidos. Además, tam- bién se entiende que las experiencias en ciencias de la salud en el uso de esta metodología está habitualmente ligada a formación práctico-clínica [2,7,9-12, 15,17-23] más que teórico-práctica, como es nuestro caso. Sin embargo, no son muchas las experiencias halladas en cuanto al manejo del contrato de aprendizaje en materias teórico-prácticas de ciencias de la salud [26], y menos aún en fisioterapia, por lo que este trabajo puede ser interesante de cara a mostrar una experiencia a partir de la cual seguir investigando.

Otra posible debilidad fueron las dudas iniciales por nuestra parte de lo positivo que podría resultar la implementación de esta estrategia de aprendizaje debido a la falta de experiencia [20]. No fue exactamente ansiedad lo que sentimos, como señalan otros estudios [10,19-21], pero sí incertidumbre sobre la buena evolución del proceso a desarrollar. Dudas similares asaltaron al alumnado al inicio, cuando se les planteó la propuesta, quizás debido a la falta de experiencia en esta metodología o al hecho de que les obligaba, de partida, a un mayor trabajo por su parte, lo que no ocurriría con la 'comodidad' del modelo docente tradicional.

Otras limitaciones del presente trabajo se deben al propio diseño del estudio. Además de no poder generalizar datos por ser una muestra pequeña y un estudio descriptivo transversal, este tipo de diseño favorece sesgos como el de extinción del recuerdo, por el paso de tiempo ocurrido desde la finalización de la materia y la valoración. Tampoco permite conocer el porqué de la no respuesta de los sujetos, pues así como puede estar causada por desidia, también puede deberse a no estar conforme con la metodología, con el proceso seguido o con la evaluación recibida. Por otra parte, el hecho de que nuestros alumnos fuesen repetidores no permite aseverar que la buena tasa de rendimiento se deba a la implementación del contrato de aprendizaje e introduce un sesgo de selección de sujetos.

Coincidimos, tanto alumnos como profesores, con lo que se indica en otros trabajos [7,10,18,20-22] sobre el esfuerzo que supone implementar el contrato de aprendizaje en cuanto a consumo de tiempo. Diseñar la experiencia, desarrollar los materiales, organizar el proceso a seguir, negociar con el alumnado el contenido a firmar en el contrato de aprendizaje, seguir y evaluar el proceso y las evidencias generadas por el alumnado, escuchar ciertas quejas del alumnado una vez implementada esta metodología sobre el esfuerzo que el contrato de aprendizaje les exigía y ofrecerles una visión positiva del mismo, etc., supone bastante esfuerzo y exige bastante dedicación durante el proceso de enseñan- 
za-aprendizaje, tanto al alumnado como al profesorado. Consideramos que el resultado final fue positivo para todos, pero también entendemos el motivo de la escasez de más experiencias en la implementación del contrato de aprendizaje dentro de materias de contenido teórico-práctico como la nuestra. Es más, teniendo en cuenta los contenidos teóricos y prácticos propios de esta materia, pensamos que si nuestra experiencia fue finalmente satisfactoria se debió, en parte, a que el alumnado no era la primera vez que se enfrentaba a dichos contenidos. Esta posibilidad de abandono de una metodología como la del contrato de aprendizaje hubiera sido bastante plausible, no sólo por lo que señalan otras investigaciones $[10,20,26,28]$ de que no gusta a determinados alumnos o no les va bien con sus formas de aprender, sino también se puede deducir por algunas de las respuestas ofrecidas por el alumnado de nuestra experiencia (pregunta 14 de la tabla II o preguntas 20 y 23 de la tabla IV). Aun así, finalmente consideramos que la experiencia fue altamente positiva tanto para el alumnado como para el profesorado.

En conclusión, el contrato de aprendizaje puede ser una de las estrategias de enseñanza a emplear para promover la participación activa de los alumnos en su propio aprendizaje, aspecto de gran importancia para facilitar en el futuro fisioterapeuta la mejora de sus competencias de cara a desarrollar un aprendizaje autónomo a lo largo de su vida profesional. Consideramos que esta experiencia ha contribuido a ello, tanto desde el punto de vista de los docentes como de los alumnos, aspecto que, además, va acorde con las líneas sugeridas por el proceso de convergencia de la educación superior en Europa en esta última década.

\section{Bibliografía}

1. Richardson JTE, Dawson L, Sadlo G, Jenkis V, Mcinnes J. Perceived academic quality and approaches to studying in the health professions. Med Teach 2007; 29: e108-16.

2. Bailey MA, Tuohy D. Student nurses' experiences of using a learning contract as a method of assessment. Nurs Educ Today 2009; 29: 758-62.

3. Bleakley A, Bligh J. Looking forward-looking back: aspects of the contemporary debate about teaching and learning medicine. Med Teach 2007; 29: 79-82.

4. Graffam B. Active learning in medical education: strategies for beginning implementation. Med Teach 2007; 29: 38-42.
5. Knowles MS. Using learning contracts: practical approaches to individualizing and structuring learning. San Francisco: Jossey-Bass Publisher; 1986.

6. McMichael JS, Corey JR. Contingency management in an introductory psychology course produces better learning. J Appl Behav Anal 1969; 2: 79-83.

7. Parsell G, Bligh J. Contract learning, clinical learning and clinicians. Postgrad Med J 1996; 72: 284-9.

8. Lobato-Fraile C. El estudio y trabajo autónomo del estudiante. In Miguel M, ed. Metodologías de enseñanza y aprendizaje para el desarrollo de competencias, Madrid: Alianza Universidad; 2006. p. 191-223.

9. Gibbon C. Contract learning in a clinical context: report of a case study. Nurs Educ Today 1989; 9: 264-70.

10. Chien W, Chan SW, Morrisey J. The use of learning contracts in mental health nursing clinical placement: an action research. Int J Nurs Stud 2002; 39: 685-94.

11. Rye KJB. Perceived benefits of the use of learning contracts to guide clinical education in respiratory care students. Respir Care 2008; 53: 1475-81.

12. Solomon P. Learning contracts in clinical education: evaluation by clinical supervisors. Med Teach 1992; 14: 205-10.

13. Arthurs JB. A juggling act in the classroom: managing different learning styles. Teach Learn Nurs 2007; 2: 2-7.

14. Hunt A, Adamson B, Higgs J, Harris L. University education and the physiotherapy professional. Physiotherapy 1998; 84: 264-73.

15. Murad MH, Varkey P. Self-directed learning in health professions education. Ann Acad Med Singapore 2008; 37: 580-90.

16. Gunn H, Goding L. Continuing professional development of physiotherapists based in community primary care trusts: a qualitative study investigating perceptions, experiences and outcomes. Physiotherapy 2009; 95: 209-14.

17. Richardson S. Implementing contract learning in a senior nursing practicum. J Adv Nurs 1987; 12: 201-6.

18. Donaldson I. The use of learning contracts in the clinical area. Nurs Educ Today 1992; 12: 431-6.

19. Cross V. Introducing learning contracts into physiotherapy clinical education. Physiotherapy 1996; 82: 21-7.

20. McAllister M. Learning contracts: an Australian experience. Nurs Educ Today 1996; 16: 199-205.

21. Chan SW, Chien WT. Implementing contract learning in a clinical context: report on a study. J Adv Nurs 2000; 31: 298-305.

22. Timmins F. The usefulness of learning contracts in nurse education: the Irish perspective. Nurse Educ Pract 2002; 2: 190-6.

23. Lacasse M, Lee S, Ghavam-Rassoul A, Batty HP. Integrating teaching into the busy resident schedule: a learner-centered approach to raise efficiency (L-CARE) in clinical teaching. Med Teach 2009; 31: e507-13.

24. Anderson G, Boud D, Sampson J. Learning contracts. A practical guide. London: Kogan Page; 1996.

25. Espey E, Ogburn T, Kalishman S, Zsemlye M, Cosgrove E. Revitalizing problem based learning: student and tutor attitudes towards a structured tutorial. Med Teach 2007; 29: 143-9.

26. Aracil-Marco A, Gallar-Martínez J. Implantación de un contrato conductual en estudiantes universitarios: una experiencia en la asignatura 'Estructura y función del cuerpo humano'. Educ Med 2008; 11: 239-46.

27. Cerezo-Bautista AM, Hernández-Álvarez J. Formando docentes, una experiencia desde el constructivismo. Aquichan 2008; 8: 64-73.

28. Smedley A. The self-directed learning readiness of first year bachelor of nursing students. J Res Nurs 2007; 12: 373-85. 